\title{
Managing Perioperative Pain After Anterior Cruciate Ligament (ACL) Reconstruction: Perspectives from a Sports Medicine Surgeon
}

\author{
loanna K Bolia $(\mathbb{D}$ \\ Aryan Haratian \\ Jennifer A Bell \\ Laith K Hasan \\ Nima Saboori \\ Ryan Palmer \\ Frank A Petrigliano \\ Alexander E Weber \\ USC Epstein Family Center for Sports \\ Medicine at Keck Medicine of USC, Los \\ Angeles, CA, USA
}

Correspondence: Alexander E Weber USC Epstein Family Center for Sports Medicine at Keck Medicine of USC, 1520 San Pablo Street, Suite 2000, Los Angeles, CA, 90033, USA

Email weber.ae@gmail.com

\begin{abstract}
Anterior cruciate ligament reconstructions (ACLR) are a relatively common procedure in orthopedic sports medicine with an estimated 130,000 arthroscopic operations performed annually. Most procedures are carried out on an outpatient basis, and though success rates of ACLR are as high as $95 \%$, pain remains the most common postoperative complication delaying patient discharge, and thereby increasing the costs associated with patient care. Despite the success and relative frequency of ACLR surgery, optimal and widely accepted strategies and regimens for controlling perioperative pain are not well established. In recent years, the paradigm of pain control has shifted from exclusively utilizing opiates and opioid medications in the acute postoperative period to employing other agents and techniques including nerve blocks, intra-articular and periarticular injections of local anesthetic agents, NSAIDs, and less commonly, ketamine, tranexamic acid (TXA), sedatives, gabapentin, and corticosteroids. More often, these agents are now used in combination and in synergy with one another as part of a multimodal approach to pain management in ACLR, with the goal of reducing postoperative pain, opioid consumption, and the incidence of delayed hospital discharge. The purpose of this review is to consolidate current literature on various agents involved in the management of postoperative pain following ACLR, including the role of classically used opiate and opioid medications, as well as to describe other drugs currently utilized in practice either individually or in conjunction with other agents as part of a multimodal regimen in pain management in ACLR.
\end{abstract}

Keywords: anterior cruciate ligament reconstruction, ACL, postoperative pain, patient outcomes, multimodal pain management

\section{Introduction}

Anterior cruciate ligament tears are one of the most common sports injuries in the United States. ${ }^{1}$ Anterior cruciate ligament reconstruction (ACLR) is the gold standard in treatment, with an estimated 130,000 arthroscopic procedures performed annually. ${ }^{2}$ Most procedures are carried out on an outpatient basis, and though success rates of ACLR are as high as 95\%, pain remains the most common postoperative complication delaying patient discharge, and thereby increasing the costs associated with patient care. ${ }^{2,3}$ Compared across other orthopedic sports medicine procedures including rotator cuff repair, meniscal debridement, and labral repair, ACLR has subjectively been ranked as the most painful. ${ }^{4}$

Despite the success and relative frequency of ACLR surgery, optimal and widely accepted strategies and regimens for controlling perioperative pain are not 
well established. ${ }^{5}$ Since successful management of postoperative pain is often correlated with improved patient outcomes, developing an effective strategy to provide adequate pain relief is of importance to orthopedic surgeons. ${ }^{6}$ Regimens in postoperative pain management after ACLR vary significantly and range from agents used either individually or given in conjunction with other medications. Common agents in current practice include narcotics, nonsteroidal anti-inflammatory agents (NSAIDs), and anesthetic agents such as lidocaine or bupivacaine that have nerve blocking and regional anesthetic capabilities. Less commonly used agents include ketamine, tranexamic acid (TXA), sedatives, gabapentinoids, and corticosteroids. ${ }^{7}$

While historically, opiates and opioids were the gold standard in postoperative pain control, complications associated with prolonged narcotic use have shifted the paradigm of pain management in orthopedic practice towards a greater focus on optimizing multimodal pain control regimens to reduce the role of narcotics. ${ }^{8-10}$ The purpose of this review is to consolidate current literature on various agents involved in the management of postoperative pain following ACLR, including the role of classically used opiate and opioid medications, as well as to describe other drugs currently utilized in practice either individually or in conjunction with other agents as part of a multimodal regimen in pain management after ACLR.

\section{Opiates and Opioids}

Opiates and their synthetic analogs, opioids, were among the most popular pain control modalities used in the acute postoperative period for a variety of orthopedic procedures. Medications in this class are thought to act on the central nervous system as agonists of the $\mathrm{Mu}$ receptor. While opioids were previously employed in greater frequency as a main pain control measure after ACLR, their associated addictiveness, abuse potential, withdrawal symptoms upon discontinuation after prolonged use, and other unpleasant side effects such as decreased bowel motility have shifted the paradigm of pain management in orthopedic surgery in recent years to a more multimodal approach. ${ }^{9-11}$ Additionally, other studies have implicated the use of opioids in delaying hospital discharge after ACLR likely leading to increased cost of care, and thus emphasizing the need to incorporate non-narcotic measures in the postoperative period. ${ }^{12}$ Nonetheless, opiate medications are still frequently employed as a measure to treat breakthrough pain within the first week after ACLR surgery, especially when patient pain is not successfully managed through alternative means. ${ }^{13}$ The most commonly prescribed opioids after ACLR include oxycodone, hydrocodone, tramadol, and less commonly, morphine. ${ }^{14}$

Various routes of administration for opioid medications in ACLR have been discussed in literature including oral, intra-articular (IA), intravenous (IV), and intrathecal. ${ }^{15-17}$ While opioids were originally thought to predominantly exhibit a centrally acting mechanism of analgesia, more recent studies with IA opioids have suggested the existence of a peripherally acting mechanism as well. ${ }^{16,17}$ A study by Brandsson et al conducted a randomized controlled trial (RCT) in patients undergoing ACLR comparing IV and IA morphine. ${ }^{16}$ Upon comparison, visual analog scale (VAS) pain scores were noted to be equivalent between IA and IV morphine, with no differences in terms of additional analgesic usage, complications, or side effects between the two groups. A different study compared use of intrathecal morphine, IA morphine, IA levobupivacaine in patients undergoing ACLR, measuring outcomes using numeric pain scales from 1 to $5 .{ }^{17}$ While pain outcomes were similar in the first 12 hours postoperatively, patients given a dose of $5 \mathrm{mg}$ IA morphine and those given $75 \mathrm{mcg}$ of intrathecal morphine had significantly less pain 24 hours postoperatively than those given either purely IA levobupivacaine or a combination of IA levobupivacaine and $2.5 \mathrm{mg}$ of IA morphine. ${ }^{17}$ A study by Karlsson et al compared use of IA morphine with IA bupivacaine in patients undergoing ACLR. ${ }^{18}$ Patients given IA bupivacaine had lower pain scores in the first 6 hours postoperatively, while those given IA morphine had sustained pain relief, and significantly lower than the bupivacaine group up to 48 hours postoperatively. While studies have shown the effectiveness of IA opioid administration in pain management after ACLR, opioids are now often administered IA in conjunction with a local anesthetic agent in an effort to provide longer lasting pain relief, and concurrently reduce the total dose of opioid medication need. ${ }^{19-22}$ Nonetheless, the success of IA opioid medications in providing pain relief in the acute postoperative period suggests a significant peripherally acting analgesic property of opioids and provides sports medicine surgeons with alternatives to intravenous and oral opioid medications, although future studies will need to explore whether opiates and opioids given IA are less likely to cause reliance or other negative side effects commonly associated with narcotic medications. 
Previous studies have attempted to quantify the degree of pain and subsequent opioid requirement based on the specific surgical technique employed in ACLR. Okoroha et al compared patient pain and total opioid requirement after either ACLR with a bone-patellar tendon-bone (BTB) graft or hamstring tendon (HT) autograft. ${ }^{23}$ The authors concluded that patients had increased acute postoperative pain when receiving BTB grafts, which subsequently led to higher incidences of breakthrough pain; however, interestingly, patients in the BTB graft group did not require a greater quantity of opioids than those receiving HT graft. ${ }^{23}$ While studies like this are not intended to guide clinical decision-making in terms of operative technique, they can provide valuable insight on which groups of patients undergoing ACLR are more likely to have a greater severity of postoperative pain, and thereby allow surgeons to better plan postoperative pain control regimens.

Several studies have explored the risk factors involved in prolonged use of opioids after ACLR surgery. ${ }^{14,24-26}$ A meta-analysis in 9474 US military personnel taking opioid medications postoperatively after ACLR revealed that $28 \%$ of the study population continued taking opioids for longer than 90 days postoperatively and identified the quantity and frequency of preoperative and perioperative opioid prescriptions filled was the largest risk factor in prolonged opioid use postoperatively. ${ }^{14}$ These results were confirmed by multiple other studies that also noted preoperative opioid usage as a major risk factor associated with prolonged opioid use postoperatively. ${ }^{24-26}$ Given the risk of patient reliance, as well as the passage of regulatory legislation limiting opioid prescriptions, the current paradigm of pain management after ACLR has shifted in recent years to a greater focus on multimodal modal regimens for pain management, with new studies and trials comparing different regimens of analgesic agents against the former standard of narcotic agents, typically seeking to quantify a regimen's effectiveness in terms of reduction in postoperative morphine consumption. . $^{8,27,28}$

\section{Local Anesthetic Agents}

Local infiltration analgesia (LIA) either intra-articular or periarticular is an effective form of anesthesia for many orthopedic procedures. Most commonly, lidocaine, bupivacaine, ketorolac and morphine are used. The use of intra-articular local anesthesia has significantly decreased postoperative pain and opioid consumption in patients who undergo ACLR. ${ }^{29}$ Many studies have also compared femoral nerve block (FNB) to intra-articular local analgesic. Two studies comparing local infiltration analgesia versus femoral nerve block in patients undergoing BTB ACLR and HT graft ACRL both found no significant difference in postoperative pain between the two cohorts. Both studies concluded that intra-articular LIA with a local anesthetic agent was sufficient for postoperative pain control if used in conjunction with a supplementary multimodal pain regimen. ${ }^{30,31}$ However, a meta-analysis by Kirkham et al reviewed 11 studies with a total of 628 patients comparing FNB to intra-articular local analgesic. ${ }^{32}$ Pain scores were found to be significantly reduced in the FNB group compared to intra-articular injection in the first two hours, 3 to 12 hours, and 13 to 24 hours postoperatively. The FNB group also had increased duration of anesthesia, decreased pain scores, and reduced consumption of IV morphine equivalents in the late postoperative period (13 to 24 hours postoperatively) compared to the intra-articular local analgesic. ${ }^{32,33}$

Periarticular infiltrations with local anesthesia can provide a safe and effective form of postoperative pain control. ${ }^{5,34}$ In a randomized control trial by Koh et al, 100 patients were randomized into 5 groups including 1) control group with no injection, 2) intra-articular (IA) ropivacaine group, 3) IA multimodal drug cocktail (MDC) 4) Periarticular (PA) injection of MDC and 5) IA + PA injection of MDC. ${ }^{5}$ PA injections were distributed to the periosteum around bone harvest sites, incision sites, patellar tendon retinaculum, infrapatellar fat pad and to the periosteum and fascia around the tibial tunnel. They found significant pain reduction in the first postoperative night in the PA MDC and IA + PA MDC, compared to the other three cohorts $(P<0.001)$. This study suggested that PA injections may be more beneficial than IA injections for perioperative pain control. Periarticular and intra-articular LIA with local anesthesia are both effective at reducing postoperative pain following ACLR and can serve a vital role in multimodal pain control regimens.

\section{Nerve Block Agents}

Anterior cruciate ligament reconstruction (ACLR) is most commonly performed in the ambulatory setting. ${ }^{2}$ As such, providing adequate pain control for timely discharge and patient satisfaction is critical to optimizing patient care and reducing unnecessary costs associated with delayed discharge. ${ }^{35}$ Regional anesthesia using peripheral nerve blocks (PNB) is increasingly implemented as perioperative pain control for orthopedic surgery procedures. PNB can 
either be given as a single-dose injection perioperatively, or catheter can be placed for continuous therapy for 3 to 5 days postoperatively. Benefits of PNB include decreased intraoperative and postoperative opioid consumption, improved postoperative pain control, and decreased fatigue and nausea that are associated with other oral forms of pain control. ${ }^{36-38}$ Side effects of PNB are rare but include small risk of nerve injury, prolonged residual motor blockade, increased risk of falls and long-lasting muscle weakness. $^{35}$

Femoral nerve block (FNB) is a commonly performed peripheral nerve block and has been considered the gold standard for the last two decades for ACLR. Two randomized control trials found a statistically significant decrease in oral morphine equivalent consumption in the first 24 hours postoperatively. ${ }^{37,38}$ Williams et al evaluated 233 patients who underwent ACLR and were randomized to a 7-day femoral nerve catheter with either 1) saline bolus and saline infusion 2) levobupivacaine $(0.25 \%)$ bolus and saline infusion or 3 ) levobupivacaine $(0.25 \%)$ bolus with levobupivacaine infusion. From postoperative day (POD) 1 through POD 4, the levobupivacaine bolus with levobupivacaine infusion has significantly lower numeric rating scale (NRS) compared to the other two groups $(P<0.001)$. The saline bolus and saline infusion group had significantly more oxycodone requirement at POD 2 and POD $3(P=0.013$ and $P=0.056) .{ }^{37}$ In a randomized control trial comparing single shock femoral nerve blocks with $25 \mathrm{~mL}$ of $0.25 \%$ bupivacaine, $25 \mathrm{~mL}$ $0.5 \%$ bupivacaine versus a sham block after ACL repair found that patients with either dosing of the bupivacaine injection had better postoperative pain control compared to the shame block. Bupivacaine $0.25 \%$ and $0.5 \%$ FNB provided 23.2 and 25.7 hours of analgesia, respectively. ${ }^{39}$ However, when FNB is used with other modes of analgesia, FNB is not found to add additional pain control. In a randomized control trial by Matava et al, patient who underwent ACLR received either FNB or placebo with saline infusion. Intra-operatively, each patient received an intra-articular bupivacaine injection and IV ketorolac. They found no significant difference between postoperative pain scores, perioperative opioid consumption, time to discharge or patient satisfaction.

Other types of PNB commonly include sciatic or adductor canal PNB. More recently in the literature, there has been concern over the risk of prolonged motor weakness of the quadriceps muscles after femoral nerve block. $^{40,41}$ Several studies have shown quadricep weakness after FNB which can result in delayed mobilization, increased recovery times or even higher risk of reinjury. ${ }^{42,43}$ Due to those concerns, adductor canal blocks (ACB) have more recently been used as an alternative due to the decreased risk of weakness to the quadriceps. An ACB is deposited to the middle third of the thigh to target the saphenous nerve, which is mostly a sensory nerve, and therefore can provide pain relief without causing muscle weakness. In a systematic review, Smith et al evaluated 5 randomized control trials with a total of 441 patients who underwent ACLR, 221 patients who received FNB and 221 patients who received ACB. ${ }^{42}$ Three studies reported greater analgesic use in the first 24 hours postoperatively in patients who received $\mathrm{ABC}$ vs FNB, ${ }^{44-46}$ however, only one paper found statistical significance. ${ }^{44}$ All but one study reported no difference in postoperative pain scores, and one reported significantly greater VAS scores in the ACB group compared to FBC. ${ }^{44}$ All studies evaluated quadricep muscle function after block and found decreased strength in the FBC versus ACB group. One study reported postoperative outcomes at 24 hours, 2 weeks, 4 weeks, and 6 months postoperatively in 90 patients who underweight ACLR with patellar tendon autograft with either ACB or $\mathrm{FNB}^{45}$ They reported no difference between subjective pain or morphine equivalent consumption. Quadriceps electromyography deficits were higher in FBC cohort at both 24 hours $(\mathrm{P}<0.001)$ and 2 weeks $(\mathrm{P}<$ 0.001). At 4 weeks postoperatively, more patients in the ACB met full ambulation criteria. ACB is an alternative to FNB that provides similar postoperative pain control and avoids weakness to the quadriceps.

\section{Non-Steroidal Anti-Inflammatory Drugs (NSAIDs)}

Non-steroidal anti-inflammatory drugs (NSAIDs) are routinely used as an analgesic agent in orthopaedic care and regularly utilized in multimodal postoperative pain management protocols. ${ }^{4,47,48}$ Numerous studies have investigated its efficacy as a postoperative analgesic and demonstrated it as an effective and safe method of treatment. ${ }^{47,49-51}$ Commonly used NSAIDs include diclofenac, ketorolac, celecoxib, and ibuprofen. ${ }^{52}$ In a systematic review of randomized control trials studying pain management following outpatient ACLR, Secrist et al found that ketorolac and ibuprofen were both effective in minimizing postoperative pain and reducing opioid consumption among patients. ${ }^{53}$ Onda et al conducted 
a randomized, parallel-group trial comparing the effects of treatment with celecoxib, loxoprofen, and acetaminophen on postoperative acute pain after arthroscopic knee surgery and found that celecoxib and loxoprofen treatments were superior to acetaminophen in pain-relief. ${ }^{54}$ In a tripleblinded randomized control trial, Mardani-Kivi et al found that the use of celecoxib as a pre-emptive analgesia after ACLR reduced pain intensity and opioid consumption in the celecoxib group at 6 and $24 \mathrm{~h}$ post operation (P $<0.0001) .{ }^{55}$ Furthermore, NSAIDs are associated with less side effects such as nausea and vomiting and improved tolerance among patients when compared to other commonly used postoperative pain medications. ${ }^{4,53,56}$ Past studies have also described how the combination of NSAIDs with other treatment modalities often yields superior results in pain control in the immediate postoperative period. ${ }^{49,51}$ Therefore, the combination of NSAIDs with other treatment modalities should be considered as a viable and effective postoperative pain management.

Past studies have suggested that the use of NSAIDs may have adverse effects on recovery and healing follow orthopaedic procedures. ${ }^{57-61}$ Furthermore, studies have revealed that celecoxib specifically can negatively affect tendon-to-bone healing follow rotator cuff surgery, causing some hesitancy in its use as a postoperative pain medication. ${ }^{59,60} \mathrm{~A}$ randomized control trial investigating whether selective COX-2 inhibitors, such as celecoxib, impact healing after arthroscopic rotator cuff repair found that retear rate in the celecoxib group (11/30 [37\%]) was significantly higher than those in the ibuprofen $(2 / 27$ [7\%]) and tramadol $(1 / 25[4 \%])$ groups $(\mathrm{P}=0.009) .{ }^{60}$ However, other studies have observed no negative effect with the use of NSAIDs and selective COX-2 inhibitors on soft tissue and tendon healing. ${ }^{62,63}$ Studies investigating the use of NSAIDs in ACL, shoulder/labrum, and meniscus repair also found no differences in healing with the use of NSAIDs postoperatively. ${ }^{62-64}$ Consequently, further studies are needed to investigate the effect of NSAID use on bone and tendon healing and to further characterize the mechanism behind any potential adverse effects. Although a negative effect of selective COX-2 inhibitors has been demonstrated following rotator cuff repair, the literature suggests that the use of NSAIDs is a safe and viable option following ACLR.

\section{Ketamine}

The incorporation of ketamine into multimodal pain management following anterior cruciate ligament reconstruction has been relatively understudied. Ketamine is drug derived from phencyclidine and is thought to exhibit antagonistic action on N-methyl-D-aspartate (NMDA) receptors. ${ }^{65} \mathrm{Zhu}$ et al's prospective, randomized trial evaluated the analgesic effect of mixing ketamine with ropivacaine in an ultrasound guided, combined femoral and sciatic nerve block (CFSNB). ${ }^{66}$ Patients were randomly assigned to 3 groups, either receiving a combination of $40-\mathrm{mg}$ ketamine and $40 \mathrm{~mL}$ of $0.375 \%$ ropivacaine perineurally (RNK), $40 \mathrm{~mL}$ of $0.375 \%$ ropivacaine and IV ketamine $40 \mathrm{mg}$ (RIK), or $40 \mathrm{~mL}$ of $0.375 \%$ ropivacaine (R). Numerical rating scale pain scores were significantly lower for the RNK group compared to RIK group and R group at 20 and 24 hours postoperatively $(\mathrm{p}=0.001){ }^{66}$ Additionally, group $\mathrm{RNK}$ exhibited a longer time to first analgesic request $(p=0.014)$ in comparison to the two other groups, suggesting that perineural ketamine enhances the antinociceptive effect of CFSNB. ${ }^{66}$ No significant difference in pain scores or duration to first analgesic request was observed in group RIK and group R. ${ }^{66}$

Additional studies on the use of ketamine in ACLR pain management have produced mixed results. One randomized controlled trial (RCT) sought to assess the analgesic effect of intraoperative IV ketamine against a saline solution control. Though no differences were observed in postoperative VAS pain scores, Menigaux et al found a significantly lower amount of cumulative morphine consumption in the first 48 hours postoperatively in the ketamine groups versus the control. ${ }^{67}$ However, these results conflicted with two other RCTs, which found that intraoperative ketamine infusion did not potentiate the analgesic effects of $0.1 \%$ ropivacaine or reduce post-surgical morphine consumption and VAS pain scores in comparison to controls. ${ }^{68,69}$

\section{Tranexamic Acid (TXA)}

Tranexamic acid (TXA) has shown promise reducing the severity of pain and incidence of hemarthrosis in the early postoperative period after ACLR. A synthetic, antifibrinolytic derivative of lysine, TXA blocks the lysine-binding site of plasminogen, competitively inhibiting fibrinolysis, and stabilizing clot formation. ${ }^{70}$ While various dosing regimens for IV TXA exist in current practice, recent studies have reported that desired hemostatic and analgesic effects 
are achieved using both 10 and $15 \mathrm{mg} / \mathrm{kg}$ doses. ${ }^{71-74}$ Both IA and IV administration have been shown to significantly decrease pain and knee joint effusion volume all without increasing the risk of thromboembolic events. ${ }^{71-74}$ Additionally, IA and IV administration are comparable in terms of efficacy with similar VAS scores at 1 week and 2 weeks postoperatively. ${ }^{74}$

Hemarthrosis is one of the major complications following ACLR, estimated to occur in $3-10 \%$ of cases. ${ }^{75}$ Given its hemostatic properties, TXA appears to be a viable solution for reducing the risk of hemarthrosis, thereby indirectly preventing a patient from experiencing pain associated with hemarthrosis formation. ${ }^{75}$ Given the relative safety and effectiveness of TXA in preventing hemarthrosis, its addition to a multimodal pain regimen has the potential of lowering a patient's pain demand and thereby reduce the need for supplemental narcotic medications.

\section{Sedatives, Gabapentinoids, Corticosteroids}

Although employed less frequently in clinical practice, sedatives, gabapentin, and corticosteroids have been explored as alternative means of pain management in ACLR. Sedatives described in the management of postoperative pain after ACLR, are typically of the nonbenzodiazepine class including agents such as zolpidem that increase the activity of inhibitory gammaaminobutyric acid (GABA) receptors centrally, and $\alpha 2$-adrenergic agonistic agents such as dexmedetomidine. ${ }^{76-78}$ Patients receiving $10 \mathrm{mg}$ of zolpidem nightly for the first week postoperatively after ACLR have been shown to have a $28 \%$ reduction in opioid consumption. ${ }^{77}$ Similarly, a study explored the use of IA dexmedetomidine when used in conjunction with levobupivacaine, versus IA levobupivacaine alone and concluded that those treated with a combination of dexmedetomidine and levobupivacaine had significantly lower opiate consumption, VAS pain scores, and required less analgesics overall in the postoperative period. ${ }^{76}$

Gabapentin and pregabalin have been utilized in medicine as both anticonvulsant and analgesic agents, especially employed in cases where pain is thought to be neuropathic in origin and are thought to exhibit their effect through modulation of various ion channels. ${ }^{79,80}$ Gabapentin administered preoperatively has been studied as potential agent in multimodal pain management after ACLR; however, studies have been mixed regarding its potential to decrease pain scores and postoperative opioid consumption. ${ }^{81,82}$ Similar RCTs have been conducted with the use of $150 \mathrm{mg}$ of preoperative and postoperative pregabalin and have demonstrated reduced subjective pain scores and postoperative opioid consumption. ${ }^{83,84}$ However, these results were in conflict with another RCT involving the use of preoperative and postoperative pregabalin in ACLR that indicated the drug no better than placebo in regard to reducing postoperative pain and opioid consumption. ${ }^{85}$ That said, it is important to note that patients in this study were only given $75 \mathrm{mg}$ of pregabalin both before and after surgery, thus possibly indicating that the dose may not have been sufficient to observe a benefit in terms of patient outcomes. ${ }^{85}$ Larger RCTs, likely with different dosing regimens, are needed to better elucidate the role of gabapentin and pregabalin in multimodal pain management after ACLR.

Given the risk of immune suppression and other side effects, the role of corticosteroids as a pain control modality is relatively limited in orthopedic surgery. Use of NSAIDs in conjunction with $8 \mathrm{mg}$ of IV dexamethasone postoperatively after ACLR has been shown to be superior in reducing pain than NSAIDs alone. ${ }^{49}$ Furthermore, the addition of 1-4 mg of dexamethasone to the solution of bupivacaine when performing a sub-sartorial saphenous nerve block (SSNB) has been shown to potentially increase the duration of the nerve block by up to 13 hours. ${ }^{86}$

\section{Multimodal Pain Management Regimens in ACLR}

With the decreased utilization of narcotic class medications and a shift in the paradigm of pain to a multimodal approach, multiple combinations and regiments of analgesic agents have been developed and trialed, with various regimens already covered in this review. A relatively recent multicenter study compared multiple non-narcotic agents and approaches in pain management after ACLR in terms of subjective pain measured through a VAS, opioid consumption, rates of delayed discharge, and complications. ${ }^{12}$ The analgesic procedures included were continuous and single dose femoral nerve blockades, and either periarticular or intra-articular LIA analgesia; all procedures were done with an unspecified locally acting anesthesia agents. ${ }^{12}$ Additionally, use of NSAIDS and IV corticosteroids was also compared. Patients receiving NSAIDs were less likely to have a delay in discharge, 
and use of dexamethasone was associated with both decreased opioid consumption and fewer side effects from nerve block agents. ${ }^{12}$ All techniques involving local anesthetic agents had comparable results in terms of postoperative pain control. The authors of the study recommended use of dexamethasone during induction preoperatively, either periarticular local infiltration anesthesia or saphenous nerve blockades, and NSAIDs for optimal management of pain after ACLR. ${ }^{12}$ A similar study by Moutzouros et al evaluated a multimodal regimen in various orthopedic sports surgical procedures including ACLR. ${ }^{4}$ The pain regimen consisted of a combination of oral preoperative celecoxib, acetaminophen, gabapentin, tramadol, and dexamethasone, as well as a femoral nerve block. This was followed by intraoperative LIA with ropivacaine. ${ }^{4}$ Postoperatively, the authors utilized ketorolac, diazepam, gabapentin, and acetaminophen in the early postoperative period (1-5 days) followed by a combination of diazepam, acetaminophen, and meloxicam in the later postoperative period (6-14 days). ${ }^{4}$ Of the patients receiving ACLR while on this regimen, over a third (38.8\%) did not require any additional opioids postoperatively for breakthrough pain while on this regimen.

\section{Conclusion}

The optimal management of postoperative pain after ACLR continues to remain a controversial topic with no widely accepted approach. In recent years, the paradigm of pain control has shifted from prescribing exclusively opiates and opioid medications to other agents and techniques including nerve blocks, intra-articular and periarticular injections of local anesthetic agents, NSAIDs, and less commonly ketamine, TXA, sedatives, gabapentin, and corticosteroids. More often, these agents are now used in combination and synergy with one another as part of a multimodal approach to pain management in ACLR, to provide synergy and thereby reduce postoperative pain, opioid consumption, and the incidence of delayed hospital discharge. While no single regimen is widely accepted or proven to be superior, future larger randomized controlled trials can directly compare the relative effectiveness across protocols to better guide surgeons in clinical decision-making with regard to ACLR pain management.

\section{Disclosure}

The authors report no conflicts of interest in this work.

\section{References}

1. Di Benedetto P, Di Benedetto E, Fiocchi A, Beltrame A, Causero A. Causes of failure of anterior cruciate ligament reconstruction and revision surgical strategies. Knee Surg Relat Res. 2016;28 (4):319-324. doi:10.5792/ksrr.16.007

2. Jansson H, Narvy SJ, Mehran N. Perioperative pain management strategies for anterior cruciate ligament reconstruction. JBJS Rev. 2018;6(3):e3. doi:10.2106/JBJS.RVW.17.00059

3. de Sa D, Shanmugaraj A, Weidman M, et al. All-inside anterior cruciate ligament reconstruction-a systematic review of techniques, outcomes, and complications. J Knee Surg. 2018;31(9):895-904. doi:10.1055/s-0038-1627446

4. Moutzouros V, Jildeh TR, Khalil LS, et al. A Multimodal protocol to diminish pain following common orthopedic sports procedures: can we eliminate postoperative opioids? Arthroscopy. 2020;36 (8):2249-2257. doi:10.1016/j.arthro.2020.04.018

5. Koh IJ, Chang CB, Seo ES, Kim SJ, Seong SC, Kim TK. Pain management by periarticular multimodal drug injection after anterior cruciate ligament reconstruction: a randomized, controlled study. Arthroscopy. 2012;28(5):649-657. doi:10.1016/j.arthro.2011.10.015

6. Chmielewski TL, Jones D, Day T, Tillman SM, Lentz TA, George SZ. The association of pain and fear of movement/reinjury with function during anterior cruciate ligament reconstruction rehabilitation. J Orthop Sports Phys Ther. 2008;38(12):746-753. doi:10.2519/jospt.2008.2887

7. Davey MS, Hurley ET, Anil U, et al. Pain Management strategies after anterior cruciate ligament reconstruction: a systematic review with network meta-analysis. Arthroscopy. 2021;37(4):1290-1300. e1296. doi:10.1016/j.arthro.2021.01.023

8. Malige A, Bram JT, Maguire KJ, McNeely LW, Ganley TJ, Williams BA. Decreased prescribing of postoperative opioids in pediatric ACL reconstruction: treatment trends at a single center. Orthop J Sports Med. 2021;9(2):2325967120979993. doi:10.1177/ 2325967120979993

9. Trasolini NA, McKnight BM, Dorr LD, Opioid T. Crisis and the Orthopedic surgeon. J Arthroplasty. 2018;33(11):3379-3382.e3371. doi:10.1016/j.arth.2018.07.002

10. Morris BJ, Mir HR. The opioid epidemic: impact on orthopaedic surgery. JAAOS. 2015;23(5):267-271. doi:10.5435/JAAOS-D-14-00163

11. Soffin EM, Waldman SA, Stack RJ, Liguori GA. An evidence-based approach to the prescription opioid epidemic in orthopedic surgery. Anesth Analg. 2017;125(5):1704-1713. doi:10.1213/ANE.0000000 000002433

12. Baverel L, Cucurulo T, Lutz C, et al. Anesthesia and analgesia methods for outpatient anterior cruciate ligament reconstruction. Orthop Traumatol Surg Res. 2016;102(8s):S251-s255. doi:10.1016/j.otsr.2016.08.007

13. Beck JJ, Cline K, Sangiorgio S, Serpa R, Shifflett KA, Bowen RE. Prospective Study of acute opioid use after adolescent anterior cruciate ligament reconstruction shows no effect from patient- or surgical-related factors. $J$ Am Acad Orthop Surg. 2020;28 (7):293-300. doi:10.5435/JAAOS-D-18-00766

14. Anderson AB, Balazs GC, Brooks DI, Potter BK, Forsberg JA, Dickens JF. Prescription patterns and risk factors for prolonged opioid dependence in elective anterior cruciate ligament reconstruction in a military population. Orthop J Sports Med. 2020;8 (6):2325967120926489. doi:10.1177/2325967120926489

15. Yari M, Saeb M, Golfam P, Makhloogh Z. Analgesic efficacy of intra-articular morphine after arthroscopic knee surgery in sport injury patients. J Inj Violence Res. 2013;5(2):84-88. doi:10.5249/ jivr.v5i2.303

16. Brandsson S, Karlsson J, Morberg P, Rydgren B, Eriksson BI, Hedner T. Intraarticular morphine after arthroscopic ACL reconstruction: a double-blind placebo-controlled study of 40 patients. Acta Orthop Scand. 2000;71(3):280-285. doi:10.1080/0001647 00317411889 
17. Pinheiro LQ, Neri Junior E, Fernandes RM, Cardozo RT, Rezende PR. Reconstruction of the anterior cruciate ligament: comparison of analgesia using intrathecal morphine, intra-articular morphine and intra-articular levobupivacaine. Rev Bras Ortop. 2015;50 (3):300-304. doi:10.1016/j.rbo.2014.04.005

18. Karlsson J, Rydgren B, Eriksson B, et al. Postoperative analgesic effects of intra-articular bupivacaine and morphine after arthroscopic cruciate ligament surgery. Knee Surg Sports Traumatol Arthrosc. 1995;3(1):55-59. doi:10.1007/BF01553527

19. Wang X, Jia D, Chen X, Xu Y. Comparison of intra-articular low-dose sufentanil, ropivacaine, and combined sufentanil and ropivacaine on post-operative analgesia of isolated anterior cruciate ligament reconstruction. Knee Surg Sports Traumatol Arthrosc. 2013;21 (5):1140-1145. doi:10.1007/s00167-012-2326-6

20. Hosseini H, Abrisham SM, Jomeh H, Kermani-Alghoraishi M, Ghahramani R, Mozayan MR. The comparison of intraarticular morphine-bupivacaine and tramadol-bupivacaine in postoperative analgesia after arthroscopic anterior cruciate ligament reconstruction. Knee Surg Sports Traumatol Arthrosc. 2012;20 (9):1839-1844. doi:10.1007/s00167-011-1791-7

21. Senthilkumaran S, Tate R, Read JR, Sutherland AG. Intra-articular morphine and bupivicaine for post-operative analgesia in anterior cruciate ligament reconstruction: a prospective randomised controlled trial. Knee Surg Sports Traumatol Arthrosc. 2010;18(6):731-735. doi:10.1007/s00167-009-0912-z

22. Mitchell BC, Siow MY, Pennock AT, Edmonds EW, Bastrom TP, Chambers HG. Intra-articular morphine and ropivacaine injection provides efficacious analgesia as compared with femoral nerve block in the first 24 hours after ACL reconstruction: results from a bone-patellar tendon-bone graft in an adolescent population. Orthop J Sports Med. 2021;9(3):2325967120985902. doi:10.1177/2325967120985902

23. Okoroha KR, Keller RA, Jung EK, et al. Pain assessment after anterior cruciate ligament reconstruction: bone-patellar tendon-bone versus hamstring tendon autograft. Orthop J Sports Med. 2016;4 (12):2325967116674924. doi:10.1177/2325967116674924

24. Rao AG, Chan PH, Prentice HA, Paxton EW, Funahashi TT, Maletis GB. Risk factors for opioid use after anterior cruciate ligament reconstruction. Am J Sports Med. 2019;47(9):2130-2137. doi:10.1177/0363546519854754

25. Forlenza EM, Lavoie-Gagne O, Lu Y, et al. Preoperative Opioid use predicts prolonged postoperative opioid use and inferior patient outcomes following anterior cruciate ligament reconstruction. Arthroscopy. 2020;36(10):2681-2688.e2681. doi:10.1016/j. arthro.2020.06.014

26. Anthony CA, Westermann RW, Bedard N, et al. Opioid demand before and after anterior cruciate ligament reconstruction. Am J Sports Med. 2017;45(13):3098-3103. doi:10.1177/0363546517719226

27. Reid DBC, Shah KN, Shapiro BH, Ruddell JH, Akelman E, Daniels AH. Mandatory prescription limits and opioid utilization following orthopaedic surgery. J Bone Joint Surg Am. 2019;101 (10):e43. doi:10.2106/JBJS.18.00943

28. Cunningham DJ, George SZ, Lewis BD. The impact of state level public policy, prescriber education, and patient factors on opioid prescribing in elective orthopedic surgery: findings from a tertiary, academic setting. Mayo Clin Proc Innov Qual Outcomes. 2021;5 (1):23-34. doi:10.1016/j.mayocpiqo.2020.08.006

29. Tetzlaff JE, Dilger JA, Abate J, Parker RD. Preoperative intra-articular morphine and bupivacaine for pain control after outpatient arthroscopic anterior cruciate ligament reconstruction. Reg Anesth Pain Med. 1999;24(3):220-224. doi:10.1097/00115550199924030-00007

30. Kristensen PK, Pfeiffer-Jensen M, Storm JO, Thillemann TM. Local infiltration analgesia is comparable to femoral nerve block after anterior cruciate ligament reconstruction with hamstring tendon graft: a randomised controlled trial. Knee Surg Sports Traumatol Arthrosc. 2014;22(2):317-323. doi:10.1007/s00167-013-2399-x
31. Mehdi SA, Dalton DJ, Sivarajan V, Leach WJ. BTB ACL reconstruction: femoral nerve block has no advantage over intraarticular local anaesthetic infiltration. Knee Surg Sports Traumatol Arthrosc. 2004;12(3):180-183. doi:10.1007/s00167-003-0464-6

32. Kirkham KR, Grape S, Martin R, Albrecht E. Analgesic efficacy of local infiltration analgesia vs. femoral nerve block after anterior cruciate ligament reconstruction: a systematic review and meta-analysis. Anaesthesia. 2017;72(12):1542-1553. doi:10.1111/anae.14032

33. Yung EM, Brull R, Albrecht E, Joshi GP, Abdallah FW. Evidence Basis for regional anesthesia in ambulatory anterior cruciate ligament reconstruction: part III: local instillation analgesia-a systematic review and meta-analysis. Anesth Analg. 2019;128(3):426-437. doi:10.1213/ANE.0000000000002599

34. Kurosaka K, Tsukada S, Nakayama H, et al. Periarticular injection versus femoral nerve block for pain relief after anterior cruciate ligament reconstruction: a randomized controlled trial. Arthroscopy. 2018;34(1):182-188. doi:10.1016/j.arthro.2017.08.307

35. Vorobeichik L, Brull R, Joshi GP, Abdallah FW. Evidence basis for regional anesthesia in ambulatory anterior cruciate ligament reconstruction: part I-femoral nerve block. Anesth Analg. 2019;128 (1):58-65. doi:10.1213/ANE.0000000000002854

36. Min H, Ouyang Y, Chen G. Anterior cruciate ligament reconstruction with the use of adductor canal block can achieve similar pain control as femoral nerve block. Knee Surg Sports Traumatol Arthrosc. 2020;28(8):2675-2686. doi:10.1007/s00167-020-05933-6

37. Williams BA, Kentor ML, Vogt MT, et al. Reduction of verbal pain scores after anterior cruciate ligament reconstruction with 2-day continuous femoral nerve block: a randomized clinical trial. Anesthesiology. 2006;104(2):315-327. doi:10.1097/00000542-200602000-00018

38. Peng P, Claxton A, Chung F, Chan V, Miniaci A, Krishnathas A. Femoral nerve block and ketorolac in patients undergoing anterior cruciate ligament reconstruction. Can J Anaesth. 1999;46 (10):919-924. doi:10.1007/BF03013124

39. Mulroy MF, Larkin KL, Batra MS, Hodgson PS, Owens BD. Femoral nerve block with $0.25 \%$ or $0.5 \%$ bupivacaine improves postoperative analgesia following outpatient arthroscopic anterior cruciate ligament repair. Reg Anesth Pain Med. 2001;26(1):24-29. doi:10.1097/ 00115550-200101000-00007

40. Krych A, Arutyunyan G, Kuzma S, Levy B, Dahm D, Stuart M. Adverse effect of femoral nerve blockade on quadriceps strength and function after ACL reconstruction. J Knee Surg. 2015;28(1):83-88.

41. Luo TD, Ashraf A, Dahm DL, Stuart MJ, McIntosh AL. Femoral nerve block is associated with persistent strength deficits at 6 months after anterior cruciate ligament reconstruction in pediatric and adolescent patients. Am J Sports Med. 2015;43(2):331-336. doi:10.1177/ 0363546514559823

42. Smith JH, Belk JW, Kraeutler MJ, Houck DA, Scillia AJ, McCarty EC. Adductor canal versus femoral nerve block after anterior cruciate ligament reconstruction: a systematic review of level I randomized controlled trials comparing early postoperative pain, opioid requirements, and quadriceps strength. Arthroscopy. 2020;36 (7):1973-1980. doi:10.1016/j.arthro.2020.03.040

43. Everhart JS, Hughes L, Abouljoud MM, Swank K, Lewis C, Flanigan DC. Femoral nerve block at time of ACL reconstruction causes lasting quadriceps strength deficits and may increase short-term risk of re-injury. Knee Surg Sports Traumatol Arthrosc. 2020;28(6):1894-1900. doi:10.1007/s00167-019-05628-7

44. El Ahl MS. Femoral nerve block versus adductor canal block for postoperative pain control after anterior cruciate ligament reconstruction: a randomized controlled double blind study. Saudi J Anaesth. 2015;9(3):279-282. doi:10.4103/1658-354X.154708

45. Bailey L, Griffin J, Elliott M, et al. Adductor Canal nerve versus femoral nerve blockade for pain control and quadriceps function following anterior cruciate ligament reconstruction with patellar tendon autograft: a prospective randomized trial. Arthroscopy. 2019;35 (3):921-929. doi:10.1016/j.arthro.2018.10.149 
46. Ghodki PS, Shalu PS, Sardesai SP. Ultrasound-guided adductor canal block versus femoral nerve block for arthroscopic anterior cruciate ligament repair under general anesthesia. $J$ Anaesthesiol Clin Pharmacol. 2018;34(2):242-246.

47. Bourne MH. Analgesics for orthopedic postoperative pain. Am J Orthop (Belle Mead NJ). 2004;33(3):128-135.

48. Beck PR, Nho SJ, Balin J, et al. Postoperative pain management after anterior cruciate ligament reconstruction. J Knee Surg. 2004;17 (1):18-23. doi:10.1055/s-0030-1247142

49. Dahl V, Spreng UJ, Waage M, Raeder JC. Short stay and less pain after ambulatory anterior cruciate ligament (ACL) repair: COX-2 inhibitor versus glucocorticoid versus both combined. Acta Anaesthesiol Scand. 2012;56(1):95-101. doi:10.1111/j.13996576.2011.02584.x

50. McGuire DA, Sanders K, Hendricks SD. Comparison of ketorolac and opioid analgesics in postoperative ACL reconstruction outpatient pain control. Arthroscopy. 1993;9(6):653-661. doi:10.1016/S07498063(05)80501-8

51. Popp JE, Sanko WA, Sinha AK, Kaeding CC. A comparison of ketorolac tromethamine/oxycodone versus patient-controlled analgesia with morphine in anterior cruciate ligament reconstruction patients. Arthroscopy. 1998;14(8):816-819. doi:10.1016/S07498063(98)70016-7

52. Constantinescu DS, Campbell MP, Moatshe G, Vap AR. Effects of perioperative nonsteroidal anti-inflammatory drug administration on soft tissue healing: a systematic review of clinical outcomes after sports medicine orthopaedic surgery procedures. Orthopaedic j Sports Med. 2019;7(4) doi:10.1177/2325967119838873

53. Secrist ES, Freedman KB, Ciccotti MG, Mazur DW, Hammoud S. Pain Management after outpatient anterior cruciate ligament reconstruction: a systematic review of randomized controlled trials. Am $J$ Sports Med. 2016;44(9):2435-2447. doi:10.1177/036354651 5617737

54. Onda A, Ogoshi A, Itoh M, Nakagawa T, Kimura M. Comparison of the effects of treatment with celecoxib, loxoprofen, and acetaminophen on postoperative acute pain after arthroscopic knee surgery: a randomized, parallel-group trial. J Orthop Sci. 2016;21 (2):172-177. doi:10.1016/j.jos.2015.11.005

55. Mardani-Kivi M, Karimi Mobarakeh M, Haghighi M, et al. Celecoxib as a pre-emptive analgesia after arthroscopic knee surgery; a triple-blinded randomized controlled trial. Arch Orthop Trauma Surg. 2013;133(11):1561-1566. doi:10.1007/s00402-013-1852-0

56. Ge H, Liu C, Shrestha A, Wu P, Cheng B. Do nonsteroidal anti-inflammatory drugs affect tissue healing after arthroscopic anterior cruciate ligament reconstruction? Med Sci Monitor. 2018;24:6038-6043. doi:10.12659/MSM.910942

57. Tsai WC, Hsu CC, Chen CP, Chen MJ, Lin MS, Pang JH. Ibuprofen inhibition of tendon cell migration and down-regulation of paxillin expression. J Orthop Res. 2006;24(3):551-558. doi:10.1002/ jor.20069

58. Tsai WC, Tang FT, Hsu CC, Hsu YH, Pang JH, Shiue CC. Ibuprofen inhibition of tendon cell proliferation and upregulation of the cyclin kinase inhibitor p21CIP1. J Orthop Res. 2004;22(3):586-591. doi:10.1016/j.orthres.2003.10.014

59. Lu Y, Li Y, Li FL, Li X, Zhuo HW, Jiang CY. Do different cyclooxygenase inhibitors impair rotator cuff healing in a rabbit model? Chin Med J. 2015;128(17):2354-2359. doi:10.4103/0366-6999.163379

60. Oh JH, Seo HJ, Lee YH, Choi HY, Joung HY, Kim SH. Do Selective COX-2 inhibitors affect pain control and healing after arthroscopic rotator cuff repair? A Preliminary Study. Am J Sports Med. 2018;46 (3):679-686. doi:10.1177/0363546517744219

61. Li Q, Zhang Z, Cai Z. High-dose ketorolac affects adult spinal fusion: a meta-analysis of the effect of perioperative nonsteroidal anti-inflammatory drugs on spinal fusion. Spine. 2011;36(7):E461468. doi:10.1097/BRS.0b013e3181dfd163
62. Chen MR, Dragoo JL. The effect of nonsteroidal anti-inflammatory drugs on tissue healing. Knee Surg Sports Traumatol Arthrosc. 2013;21(3):540-549. doi:10.1007/s00167-012-2095-2

63. Blomquist J, Solheim E, Liavaag S, Baste V, Havelin LI. Do nonsteroidal anti-inflammatory drugs affect the outcome of arthroscopic Bankart repair? Scand J Med Sci Sports. 2014;24(6):e510-514. doi: $10.1111 /$ sms. 12233

64. Proffen BL, Nielson JH, Zurakowski D, Micheli LJ, Curtis C, Murray MM. The Effect of perioperative ketorolac on the clinical failure rate of meniscal repair. Orthop J Sports Med. 2014;2:5. doi:10.1177/2325967114529537

65. Peltoniemi MA, Hagelberg NM, Olkkola KT, Saari TI. Ketamine: a Review of clinical pharmacokinetics and pharmacodynamics in anesthesia and pain therapy. Clin Pharmacokinet. 2016;55 (9):1059-1077. doi:10.1007/s40262-016-0383-6

66. Zhu T, Gao Y, Xu X, Fu S, Lin W, Sun J. Effect of ketamine added to ropivacaine in nerve block for postoperative pain management in patients undergoing anterior cruciate ligament reconstruction: a randomized trial. Clin Ther. 2020;42(5):882-891. doi:10.1016/j. clinthera.2020.03.004

67. Menigaux C, Fletcher D, Dupont X, Guignard B, Guirimand F, Chauvin M. The benefits of intraoperative small-dose ketamine on postoperative pain after anterior cruciate ligament repair. Anesth Analg. 2000;90(1):129-135. doi:10.1097/00000539-20000100000029

68. Rahimzadeh P, Faiz SH, Ziyaeifard M, Niknam K. Effectiveness of adding ketamine to ropivacaine infusion via femoral nerve catheter after knee anterior cruciate ligament repair. J Res Med Sci. 2013;18 (8):632-636.

69. Jaksch W, Lang S, Reichhalter R, Raab G, Dann K, Fitzal S. Perioperative small-dose $\mathrm{S}(+)$-ketamine has no incremental beneficial effects on postoperative pain when standard-practice opioid infusions are used. Anesth Analg. 2002;94(4):981-986. doi:10.1097/00000539200204000-00038

70. Lin ZX, Woolf SK. Safety, efficacy, and cost-effectiveness of tranexamic acid in orthopedic surgery. Orthopedics. 2016;39 (2):119-130. doi:10.3928/01477447-20160301-05

71. Chiang ER, Chen KH, Wang ST, et al. Intra-articular injection of tranexamic acid reduced postoperative hemarthrosis in arthroscopic anterior cruciate ligament reconstruction: a prospective randomized study. Arthroscopy. 2019;35(7):2127-2132. doi:10.1016/j.arthro.201 9.02.018

72. Felli L, Revello S, Burastero G, et al. Single intravenous administration of tranexamic acid in anterior cruciate ligament reconstruction to reduce postoperative hemarthrosis and increase functional outcomes in the early phase of postoperative rehabilitation: a randomized controlled trial. Arthroscopy. 2019;35(1):149-157. doi:10.1016/j. arthro.2018.07.050

73. Karaaslan F, Karaoğlu S, Yurdakul E. Reducing Intra-articular Hemarthrosis After Arthroscopic Anterior Cruciate Ligament Reconstruction by the Administration of Intravenous Tranexamic Acid: a Prospective, Randomized Controlled Trial. Am J Sports Med. 2015;43(11):2720-2726. doi:10.1177/0363546515599629

74. Ma R, Wu M, Li Y, et al. The comparative efficacies of intravenous administration and intra-articular injection of tranexamic acid during anterior cruciate ligament reconstruction for reducing postoperative hemarthrosis: a prospective randomized study. BMC Musculoskelet Disord. 2021;22(1):114. doi:10.1186/s12891-021-03990-7

75. Bahl V, Goyal A, Jain V, Joshi D, Chaudhary D. Effect of haemarthrosis on the rehabilitation of anterior cruciate ligament reconstruction-single bundle versus double bundle. J Orthop Surg Res. 2013;8:5. doi:10.1186/1749-799X-8-5

76. Ülgey A, Bayram A, Aksu R, Altuntaş R, Güney A, Güler G. Does Treatment with Dexmedetomidine Intra-articularly Improve Postoperative Pain and Rehabilitation after Anterior Cruciate Ligament Reconstruction? J Knee Surg. 2021;2:25487. 
77. Tompkins M, Plante M, Monchik K, Fleming B, Fadale P. The use of a non-benzodiazepine hypnotic sleep-aid (Zolpidem) in patients undergoing ACL reconstruction: a randomized controlled clinical trial. Knee Surg Sports Traumatol Arthrosc. 2011;19(5):787-791. doi:10.1007/s00167-010-1368-X

78. O’Hagan ET, Hübscher M, Miller CB, et al. Zolpidem reduces pain intensity postoperatively: a systematic review and meta-analysis of the effect of hypnotic medicines on post-operative pain intensity. Syst Rev. 2020;9(1):206. doi:10.1186/s13643-020-01458-8

79. Wiffen PJ, Derry S, Bell RF, et al. Gabapentin for chronic neuropathic pain in adults. Cochrane Database Syst Rev. 2017;6(6):Cd007938.

80. Sills GJ. The mechanisms of action of gabapentin and pregabalin. Curr Opin Pharmacol. 2006;6(1):108-113. doi:10.1016/j.coph.2005.11.003

81. Ahearn B, Kumar A, Premkumar A, et al. Effect of Preoperative Gabapentin With a Concomitant Adductor Canal Block on Pain and Opioid Usage After Anterior Cruciate Ligament Reconstruction. Orthop J Sports Med. 2019;7(3):2325967119828357. doi:10.1177/ 2325967119828357

82. Mardani-Kivi M, Mobarakeh MK, Keyhani S, Motlagh KH, Ekhtiari KS. Is gabapentin effective on pain management after arthroscopic anterior cruciate ligament reconstruction? A triple blinded randomized controlled trial. Arch Bone Jt Surg. 2013;1(1):18-22.
83. Cho EA, Kim N, Lee B, Song J, Choi YS. The Effect of Perioperative Pregabalin on Pain after Arthroscopic Anterior Cruciate Ligament Reconstruction: a Randomized Controlled Trial. J Clin Med. 2019;8 (9):1426. doi:10.3390/jcm8091426

84. Kavak Akelma F, Baran Akkuş I, Altinsoy S, Özkan D, Ergil J. The effects of pregabalin and adductor canal block on postoperative pain in arthroscopic anterior cruciate ligament reconstruction. Turk J Med Sci. 2020;50(1):195-204.

85. Nimmaanrat $\mathrm{S}$, Tangtrakulwanish $\mathrm{B}$, Klabklay $\mathrm{P}$, Boonriong $\mathrm{T}$. Perioperative administration of pregabalin in patients undergoing arthroscopic anterior cruciate ligament reconstruction: does it help to relieve postoperative pain? J Med Assoc Thai. 2012;95 (10):1297-1301.

86. Chisholm MF, Cheng J, Fields KG, et al. Perineural dexamethasone with subsartorial saphenous nerve blocks in ACL reconstruction. Knee Surg Sports Traumatol Arthrosc. 2017;25(4):1298-1306. doi:10.1007/s00167-016-4120-3

\section{Publish your work in this journal}

Open Access Journal of Sports Medicine is an international, peerreviewed, open access journal publishing original research, reports, reviews and commentaries on all areas of sports medicine. The manuscript management system is completely online and includes a very quick and fair peer-review system. Visit http://www.dovepress. $\mathrm{com} /$ testimonials.php to read real quotes from published authors. 